EARLY DETECTION

\section{Making an early mark?}

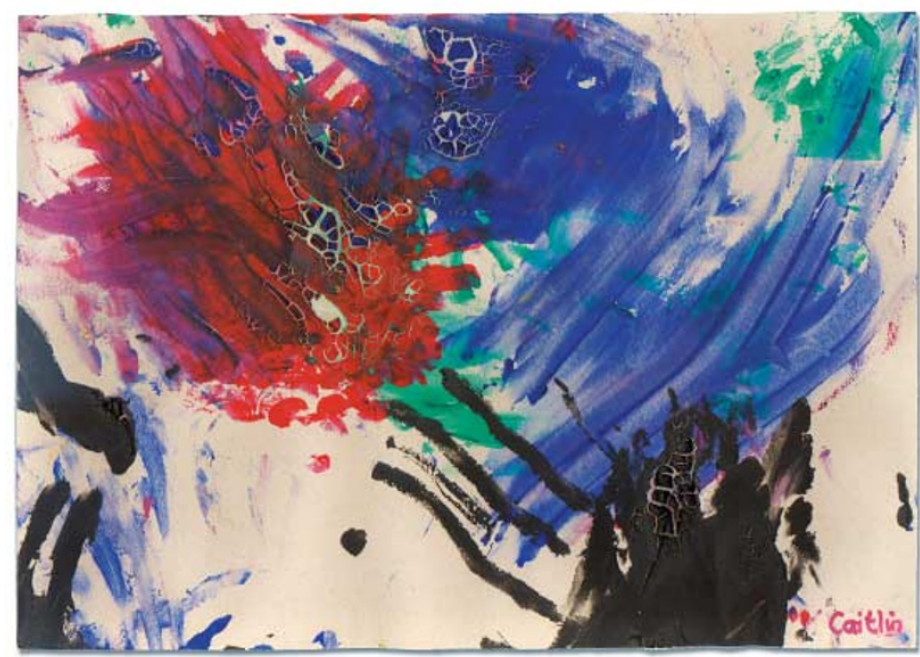

Early detection is important for patients with ovarian cancer, who, because of the late onset of clinical symptoms, are usually diagnosed with relatively advanced-stage disease. In an attempt to identify early disease markers, Ibanez de Caceres et al. have evaluated the methylation patterns of several tumoursuppressor genes.

Many genes have been shown to be hypermethylated in tumour cells and have been successfully used to identify patients with lung, head and neck, breast and bladder cancer - not directly from biopsy tissue, but in cells isolated from body fluids. This is feasible because methylated genes can be detected by a sensitive ( 1 methylated allele in 1,000 unmethylated alleles) methylation-specific PCR assay.

Ibanez de Caceres et al. used this method to determine the methylation status of two tumour-suppressor genes, BRCA1 and RASSF1A, in DNA isolated from tumour tissue, and matched serum and peritoneal fluid from 50 ovarian or primary peritoneal tumours. Sixty-eight percent of the DNA samples isolated directly from the tumour tissue showed hypermethylation of one or both genes. The authors also analysed other tumour-suppressor genes - $A P C$, CDKN2A (encoding INK4A and $\mathrm{ARF}$ ) and $D A P K$ - and increased detection of hypermethylated alleles to all 50 samples. A similar analysis of 21 archived stage I tumours revealed that $95 \%$ of samples had detectable levels of methylation at one or more of the six genes, confirming that hypermethylation is a marker of early-stage disease. Eighty-two percent of the matched serum and 93\% of peritoneal-fluid DNA showed an identical methylation pattern to that seen for the tumour DNA, indicating that body fluids, rather than tumour biopsy samples, could be analysed by this approach. Importantly, hypermethylation was not detected in tissue, serum or peritoneal-fluid DNA from 10 patients with benign ovarian disease or in serum DNA from 20 age-matched controls.

The authors concluded that analysis of the methylation pattern of these genes using serum DNA can identify individuals with ovarian cancer with $100 \%$ specificity and $82 \%$ selectivity. Improvements to the sensitivity of this approach might allow analysis of DNA hypermethylation to be developed as a non-invasive technique for identifying individuals with early-stage ovarian tumours.

Nicola McCarthy

\section{(2) References and links} ORIGINAL RESEARCH PAPER de Caceres, I.I. et al. Tumour cell specific BRCA1 and RASSF1A hypermethylation in serum, plasma and peritoneal fluid from ovarian cancer patients. Cancer Res. 64 6476-6481 (2004)

FURTHER READING Belinsky, S. Gene-promotor hypermethylation as a biomarker in lung cancer. Nature Rev. Cancer 4, 707-717 (2004)
TRIAL WATCH

\section{Predicting neuroblastoma outcome}

A gene-expression profile study, combined with analysis by artificial neural networks, has been developed to predict outcome of patients with neuroblastoma.

Fifty-six primary neuroblastoma tumour samples taken from 49 patients before treatment were retrospectively analysed by geneexpression profiling using cDNA microarrays that contained over 25,000 genes. The patients were divided into either good (eventfree survival for greater than 3 years) or poor (death due to disease) outcome groups. Neural networks - specialized pattern algorithms modelled after the human brain — were then trained to recognize or predict which samples were associated with good or poor prognosis.

The algorithm identified 19 genes, including 2 prognostic markers that were previously reported (MYCN and CD44), that could be used to predict the outcome for $98 \%$ of these patients. Several other genes known to be involved in neuronal development were also associated with outcome, revealing new therapeutic targets. Using these predictor genes, the researchers were also able to partition the subset of patients classified as highrisk into good- and poor-outcome groups after therapy, allowing them to distinguish groups of ultra-high-risk patients that are not likely to respond to conventional therapy and therefore require alternative treatment strategies.

Because only 19 genes were associated with outcome, it is possible to develop simple prognostic assays for clinical use. Three of the genes associated with poor outcome encode proteins that are secreted into the blood, so they might also be used as serum prognosis factors. The authors have begun a larger validation study that includes over 300 neuroblastoma tumour samples.

ORIGINAL RESEARCH PAPER Wei, J. S. et al. Prediction of clinical outcome using gene expression profiling and artificial neural networks for patients with neuroblastoma. Cancer Res. 64, 6883-6891 (2004)

\section{Test for bladder cancer recurrence underway}

A 3-year Phase III study to validate microsatellite DNA analysis as a method for detecting recurrent bladder cancer has been initiated by the National Cancer Institute's Early Detection Research Network (EDRN) at 12 centres across the United States and Canada.

The goal of the study is to associate microsatellite DNA sequences isolated from urine samples of 300 patients diagnosed with bladder cancer with cancer recurrence. DNA will be extracted from cells that are normally present in urine and compared with DNA sequences of unaffected cells, such as lymphocytes, from the same patients. Healthy individuals and those with non-cancerous bladder problems will be used as controls.

Early studies showed that this non-invasive procedure could identify patients with bladder cancer with over $90 \%$ accuracy. Bladder cancer has a high recurrance rate, so frequent surveillance is important. Current cytological or cytoscopic tests are often inaccurate, but this assay, if successfully validated, will provide a more sensitive and non-invasive approach. Final results are expected in September 2007, and the EDRN is also developing tests for protein biomarkers in blood serum that can be used to detect early tumours of the prostate and liver.

\section{FURTHER INFORMATION}

The Early Detection Research Network: http://www3.cancer.gov/prevention/cbrg/edrn. 